\title{
Impaired mitochondrial respiration promotes dendritic branching via the AMPK signaling pathway
}

\author{
A Gioran ${ }^{1}, \mathrm{P}$ Nicotera ${ }^{1}$ and D Bano ${ }^{*, 1}$
}

Functional neuronal circuits require a constant remodeling of their network composed of highly interconnected neurons. The plasticity of synapses and the shaping of elaborated dendritic branches are energy demanding and therefore depend on an efficient mitochondrial oxidative phosphorylation (OXPHOS). The spatial and functional regulations of dendritic patterning occur also after cell fate specification; however, the molecular mechanisms underlying this complex process remain elusive. Here, we exploit the changes in dendritic architecture in highly branched neurons as a result of aberrant mitochondrial activity. In sensory neurons of Caenorhabditis elegans, genetic manipulations of mitochondrial complex I subunits cause an unexpected outgrowth of dendritic arbors and ectopic structures. The increased number of dendritic branches is coordinated through a specific signaling cascade rather than as a simple consequence of oxidative stress. On the basis of genetic and pharmacological evidence, we show that OXPHOS deficiency promotes branching through the activation of the AMP-activated protein kinase AMPK and the downstream target phosphoinositide 3-kinase PI3K. Taken together, our findings describe a well-defined signaling pathway that regulates dendritic outgrowth in conditions of compromised OXPHOS and the resulting AMPK activation.

Cell Death and Disease (2014) 5, e1175; doi:10.1038/cddis.2014.144; published online 10 April 2014

Subject Category: Neuroscience

Mitochondria are intracellular double-membrane organelles that take part in a wide range of cellular processes, including nutrient catabolism, biosynthesis of metabolic intermediates, signaling and energy production via oxidative phosphorylation (OXPHOS). The OXPHOS system comprises the respiratory chain and the ATP synthase that generates ATP from ADP and inorganic phosphate. In the cytosol and in the mitochondrial matrix, the oxidation of fuel substrates from different carbon sources generates $\mathrm{NADH}$ and $\mathrm{FADH}_{2}$. These reduced molecules provide equivalents to the electron transport chain (ETC), consisting of four multisubunit complexes embedded in the mitochondrial inner membrane and two mobile electron carriers. ${ }^{1,2}$ Based on recent evidence, the electron flux occurs in quaternary supramolecular structures, also called respirasomes, rather than as a result of random encounters between individual ETC components. ${ }^{3,4}$ The transport of electrons to molecular oxygen as the final acceptor is coupled with the formation of a proton gradient across the membrane. This membrane potential is then dissipated by the complex V/ATP synthases for energy production or for the maintenance of other metabolic activities. Mitochondria retain evident vestiges of their original endosymbiotic prokaryotic ancestor. Apart from the highly dynamic double membrane, mitochondria contain a circular genome that encodes genes involved in mitochondrial protein translation and 13 essential subunits of the complex I, III, IV and V of the OXPHOS system. The remaining thousand or so proteins are nuclear encoded and actively imported in the mitochondria, where they need to be assembled into functionally organized complexes. ${ }^{5}$
Given their essential role in cellular metabolism, genetically inherited mutations as well as off-target effects of chemical compounds that disturb mitochondrial functions can seriously impinge healthy lifespan and inevitably lead to severe syndromes or progressive degenerative pathologies. ${ }^{2,6-8}$ From the clinical standpoint, human diseases caused by mitochondrial impairment exhibit heterogeneous manifestations that range from single tissue lesions to multiple organ failure. The vulnerability of cell types, the evoked clinical aspect and the age of onset of the disease depend on the extent of the mitochondrial damage. In eukaryotic cells, adaptive stressresponsive pathways partially respond to mitochondrial defects by promoting defensive mechanisms and alternative routes of energy production that, at least in some animal models, can ultimately result in lifespan extension. ${ }^{8,9}$ However, when the cellular damage exceeds a certain threshold, these compensatory processes are not sufficient to prevent deleterious effects on cellular maintenance, thus resulting in various diseases. As a quantitative decline of mitochondrial activity occurs during aging, faulty OXPHOS contributes to the onset of age-related human pathologies, including many common neurodegenerative diseases such as Parkinson's and Alzheimer's disease. The discovery that several inherited forms of brain disorders are due to mutations in genes involved in mitochondrial activity supports this view. ${ }^{10}$ Yet, how OXPHOS impairment can affect neuronal activity, neural branching and the plasticity of neural circuits remains an important open question. Therefore, the use of animal models carrying OXPHOS defects represents a useful tool for dissecting

\footnotetext{
${ }^{1}$ German Center for Neurodegenerative Diseases (DZNE), Bonn, Germany

*Corresponding author: D Bano, Deutsches Zentrum für Neurodegenerative Erkrankungen (DZNE), Ludwig-Erhard-Allee 2, D-53175 Bonn, Germany. Tel: +49 228 43302510; Fax: +49 228 43302689; E-mail: daniele.bano@dzne.de

Keywords: AMPK; Caenorhabditis elegans; dendritic branching; mitochondria; OXPHOS

Abbreviations: AICAR, 5-aminoimidazole-4-carboxamide ribonucleotide; AMPK, $5^{\prime}$-adenosine monophosphate-activated protein kinase; $C$. elegans, Caenorhabditis elegans; OXPHOS, oxidative phosphorylation; PI3K, phosphatidylinositol-4,5-bisphosphate 3-kinase

Received 14.2.14; accepted 27.2.14; Edited by A Verkhratsky
} 
in vivo signaling cascades with therapeutic relevance not only for inherited 'mitochondrial disorders' but also for many other human brain pathologies. ${ }^{5}$

The nematode Caenorhabditis elegans is a transparent freeliving organism with highly characterized embryonic and larval development. In the past decades, the generation and characterization of a large number of mutants have provided unique tools for extensive genetic analysis. Indeed, $C$. elegans has been widely used as a model organism for studying neural development. Despite its small size, an adult hermaphrodite has a nervous system comprising 302 neurons that control a large series of complex behavioral repertoires. ${ }^{11}$ Each neuron is classified into a group according to anatomical and functional criteria, while neural circuits have been characterized through the use of genetic mutants, electron micrographs and singlecell imaging of genetically encoded fluorescent proteins. The majority of $C$. elegans neurons are unipolar or bipolar cells with chemically or electrically defined synapses. However, the two sensory PVD neurons in the body and the two FLP neurons in the head of the animal display highly branched dendrites. ${ }^{12}$ Given their similarity with mammalian neurons and the suitability of nematodes for genetic analysis, these two classes of neurons have been extensively studied to unveil evolutionarily conserved signaling pathways involved in cell fate specification and morphogenesis.

Here, we investigated dendritic branching in animal models of OXPHOS impairment. In nematodes carrying mutations of complex I/NADH-ubiquinone oxidoreductase subunits, we unexpectedly observed an increased number of dendrites in PVD somatosensory neurons. The same changes were also recapitulated in other sensory neurons in $C$. elegans.
We studied the signaling pathways underlying these changes. On the basis of genetic and pharmacological evidence, compromised OXPHOS led to AMPK activation, with PI3K as one of the downstream targets. Taken together, we propose the AMPK/PI3K axis as a critical regulator of dendritic remodeling in conditions of compromised mitochondrial function.

\section{Results}

Complex I impairment alters dendritic structures in C. elegans sensory neurons. PVD somatosensory neurons are postembryonically generated during the L2 larval stage. The cell soma is positioned in the posterior part of the organism body, while a single axonal projection grows anteriorly and joins the ventral nerve cord. Between the second and third larval stages, PVD neurons extend longitudinally primary branches both in the posterior and anterior part of the body (Figure 1a). At adulthood, PVD neurons display secondary $\left(2^{\circ}\right)$, tertiary $\left(3^{\circ}\right)$ and quaternary $\left(4^{\circ}\right)$ side dendritic branches that envelop the animal body in a dense network of processes underneath the skin. ${ }^{12,13}$ Notably, the $2^{\circ}-3^{\circ}-4^{\circ}$ branches compose elaborate candelabra-like structural units called 'menorahs' (Figure 1b). To visualize PVD menorahs at high-resolution confocal microscopy, we used a $C$. elegans strain expressing the reporter ser-2prom3:: gfp $^{14}$ (Figures 1a and b). Normally, each animal exhibits $\sim 38$ of these candelabra-shaped structures and the number shows a constant distribution pattern in individual organisms, although over time there is a considerable decline in the maintenance of the well-organized menorah structure (data not shown). To determine whether impaired OXPHOS

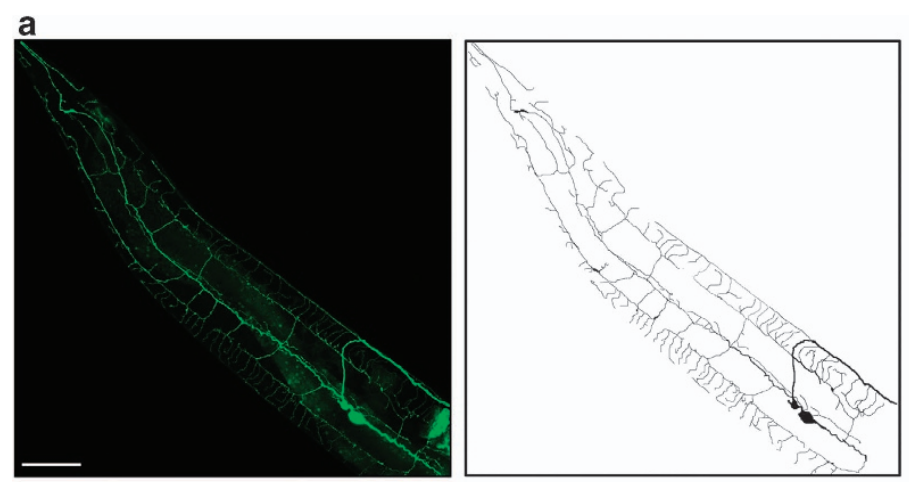

b
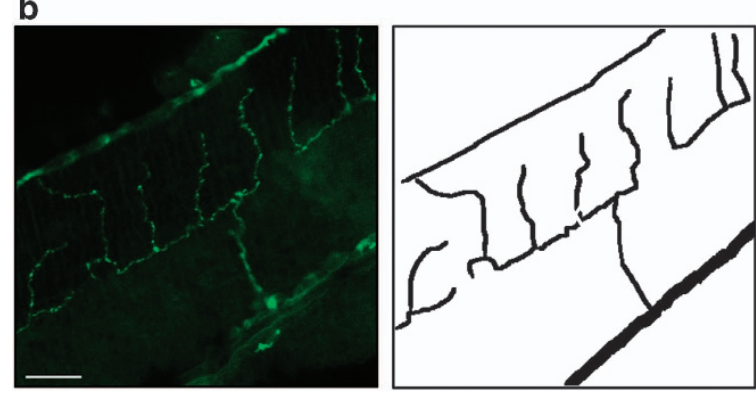

C

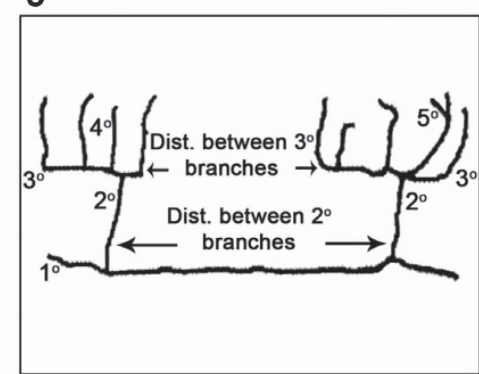

Figure 1 The PVD neuron exhibits an elaborate branching pattern. (a) Confocal image (left panel) and schematic tracing (right panel) show a PVD neuron in the posterior part of an animal. Scale bar represents $25 \mu \mathrm{m}$. (b) Confocal image (left panel) and schematic (right panel) depict at high magnification a representative menorah. Scale bar represents $10 \mu \mathrm{m}$. (c) Schematic diagram of two menorahs describes the orthogonal dendritic array as organized in $1^{\circ}, 2^{\circ}, 3^{\circ}, 4^{\circ}$ and $5^{\circ}$ branches and the distance between neighboring $2^{\circ}$ and $3^{\circ}$ branches, which are measured in this study 
can affect dendrites in vivo, we simplified our assay by scoring menorahs in the posterior part of the $C$. elegans body on the first day of adulthood. First, we assessed the number of 2 branches per $100 \mu \mathrm{m}$. Then, we measured the distance between the $2^{\circ}$ and $3^{\circ}$ branches, which can be considered the 'stem' and the 'base' of the candelabra-like structure (Figure 1c). In wild-type nematodes, more than $85 \%$ of the animals displayed approximately four $2^{\circ}$ branches per $100 \mu \mathrm{m}$ (Figures 2a and b). As a model of severe mitochondrial deficiency, we introduced the integrated ser-2prom3::gfp transgene in gas-1(fc21) loss-of-function mutant nematodes. The gas-1(fc21) is a missense mutation that affects the expression of one subunit of complex I, ${ }^{15}$ resulting in impaired mitochondrial respiration and short lifespan. We scored $2^{\circ}$ branches in gas-1 mutants and, interestingly, we observed a significantly increased number of structural units compared with wild-type nematodes (Figures $2 a$ and b). To confirm the increased density of dendritic arbors, we also measured the distance between the $2^{\circ}$ and $3^{\circ}$ branches. In agreement with our previous observation, we noticed that structural units occurred with an almost double frequency in gas-1(fc21) mutant nematodes compared with the wild-type ones (Figures $2 \mathrm{c}$ and $\mathrm{d}$ ). Thus, our in vivo analysis suggests that complex I inhibition promotes excessive branching. Furthermore, as the total length of $3^{\circ}$ branches was unaffected (Figure 2e), we can conclude that a single menorah covered a significantly smaller receptive field in mitochondrial mutants. To determine whether this phenotype occurs independently of the OXPHOS defect, we analyzed another strain overexpressing ser-2prom3::gfp and carrying a missense point mutation in the nuo- 6 gene encoding the NDUFB4/B15 subunit of complex I. ${ }^{16}$ In line with our previous evidence in gas-1 mutants, nuo- 6 mutant nematodes exhibited a significantly increased branching pattern as demonstrated by the number of $2^{\circ}$ branches and the decreased distance between them (Figures $2 f$ and $g$ ). As animals carrying OXPHOS defects exhibit enhanced oxidative stress, ${ }^{9,16}$ we assessed the number of $2^{\circ}$ branches in gas- 1 mutants exposed to the antioxidant Vitamin C. Notably, the increased dendritic branching was not affected by antioxidant treatment (Figure 2h). Next, we tested whether compromised OXPHOS affects dendritic architecture in a cell-dependent manner, and, as an additional neuronal cell type, we analyzed the two PLM neurons in a strain overexpressing the integrated $m e c-4 p:: g f p$ transgene. The PLM cells are two of the six touch receptor neurons and normally show a single process that anteriorly projects from each soma. Interestingly, whereas in control wild-type animals PLM neurons rarely showed ectopic structures, in gas-1(fc21) mutant nematodes there was a twofold increase in extra protrusions (Figures $2 \mathrm{i}$ and $\mathrm{j}$ ). Collectively, our data suggest that impaired mitochondrial ETC causes growth of extra dendritic branches in vivo in $C$. elegans, independently of the enhanced oxidative stress and regardless the genetic manipulation.

AMPK activation promotes alteration of dendritic arbors. To determine the mechanisms by which OXPHOS defects induce extra branching in nematodes, we tested potential downstream signaling pathways that might become active due to energy deprivation. Among them, AMPK is a promising candidate as it is a crucial energy sensor involved in many catabolic processes engaged during stress, including conditions of altered mitochondrial respiration. Indeed, decreased energy levels promote the binding of AMP to AMPK, causing an allosteric activation that allows the phosphorylation of Thr172 in the $\alpha$-kinase domain by upstream kinases like LKB1 or CaMKK $\beta .{ }^{17}$ In $C$. elegans, both aak-1 and aak-2 genes encode two homologs of the $\alpha$-catalytic subunit of AMPK, with aak- 2 taking part in stressresponse signaling pathways due to energy crisis. ${ }^{18,19} \mathrm{We}$ assessed the phosphorylation status of $C$. elegans AMPK and observed significantly increased levels of phosphoAMPK in homogenates from gas-1 mutants compared with wild-type animals (Figure 3a). As we did not observe any band in aak-2-null mutant nematodes, AAK-2 is likely the main phosphorylated AMPK (Figure 3a). Next, we generated gas-1; aak-2 double mutant animals overexpressing the fluorescent reporter ser-2prom3::gfp. While gas-1 loss-offunction caused consistent increased dendritic branching, neither the number of menorahs and $2^{\circ}$ branches nor the distance between $2^{\circ}$ branches was considerably different in gas-1; aak-2 double mutants compared with wild-type nematodes (Figures $3 b-d$ ). This suggests that AAK-2/AMPK is required to promote extra branching as a result of OXPHOS deficiency. In mammals, during neuronal polarization AMPK overactivation disrupts the PI3K association with kinesin Kif5 and the consequent axonal transport. Consequently, PI3K is not correctly targeted to the axonal ending and cannot sustain the proper activity of the downstream target Akt. $^{20}$ Similarly, PI3K is responsible for netrindependent axonal outgrowth during nematode development. ${ }^{21}$ To define the eventual role of PI3K in dendrite outgrowth, we generated $C$. elegans strains carrying a lossof-function allele for the age-1 gene, which encodes the p110 subunit of $\mathrm{PI} 3 \mathrm{~K} .{ }^{22}$ Consistently with previous studies, age-1 loss-of-function completely suppressed the increased dendritic branches induced by OXPHOS impairment (Figures $3 b-d)$, supporting the hypothesis that AGE-1/PI3K takes part in the process. We hypothesized that AAK-2/AMPK overactivation would mimic the condition of OXPHOS impairment and lead to a comparable enhancement of dendritic branching. Thus, we exposed nematodes to the 5-amino-imidazolecarboxamide riboside (AICAR), an AMP mimetic that directly activates AMPK and was previously suggested as a therapeutic approach in the treatment of mitochondrial disorders. ${ }^{23}$ In line with our hypothesis, AICAR stimulated AAK-2/AMPK (Figure 3e) and promoted dendritic branching in wild-type nematodes, but it did not further increase the menorah number in gas-1(fc21) animals, suggesting that the AAK-2/AMPK pathway is already engaged during OXPHOS impairment (Figure 3f). At the protein level, the decreased P-AMPK in aak-2-null mutants confirmed the specificity of AICAR (Figure 3e). Notably, AICAR did not promote dendritic branching in aak-2 mutant animals (Figure $3 \mathrm{~g}$ ). Finally, to determine whether AAK-2 acts upstream AGE-1, we hypothesized that AICAR treatment would not promote extra branching in age-1 mutants. In support of this view, we found that age-1 loss-of-function considerably suppressed the effect of AICAR (Figure 3h). This strongly suggests that AAK-2/AMPK induces dendritic branching through AGE-1/PI3K. 
a
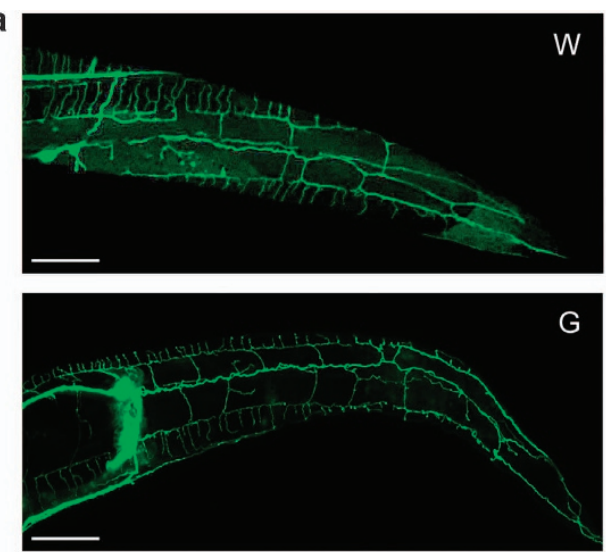

G

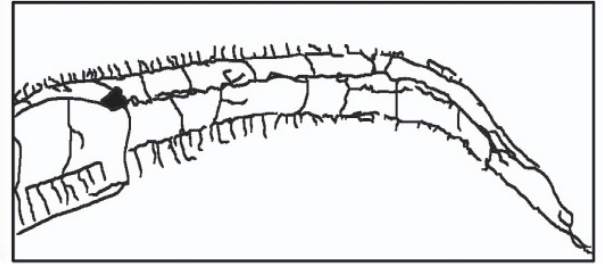

b

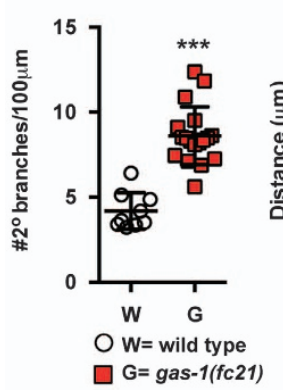

C $\quad 2^{\circ}$ branches

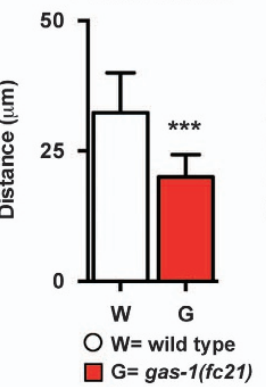

f

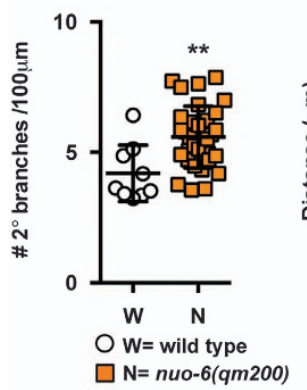

g

$2^{\circ}$ branches
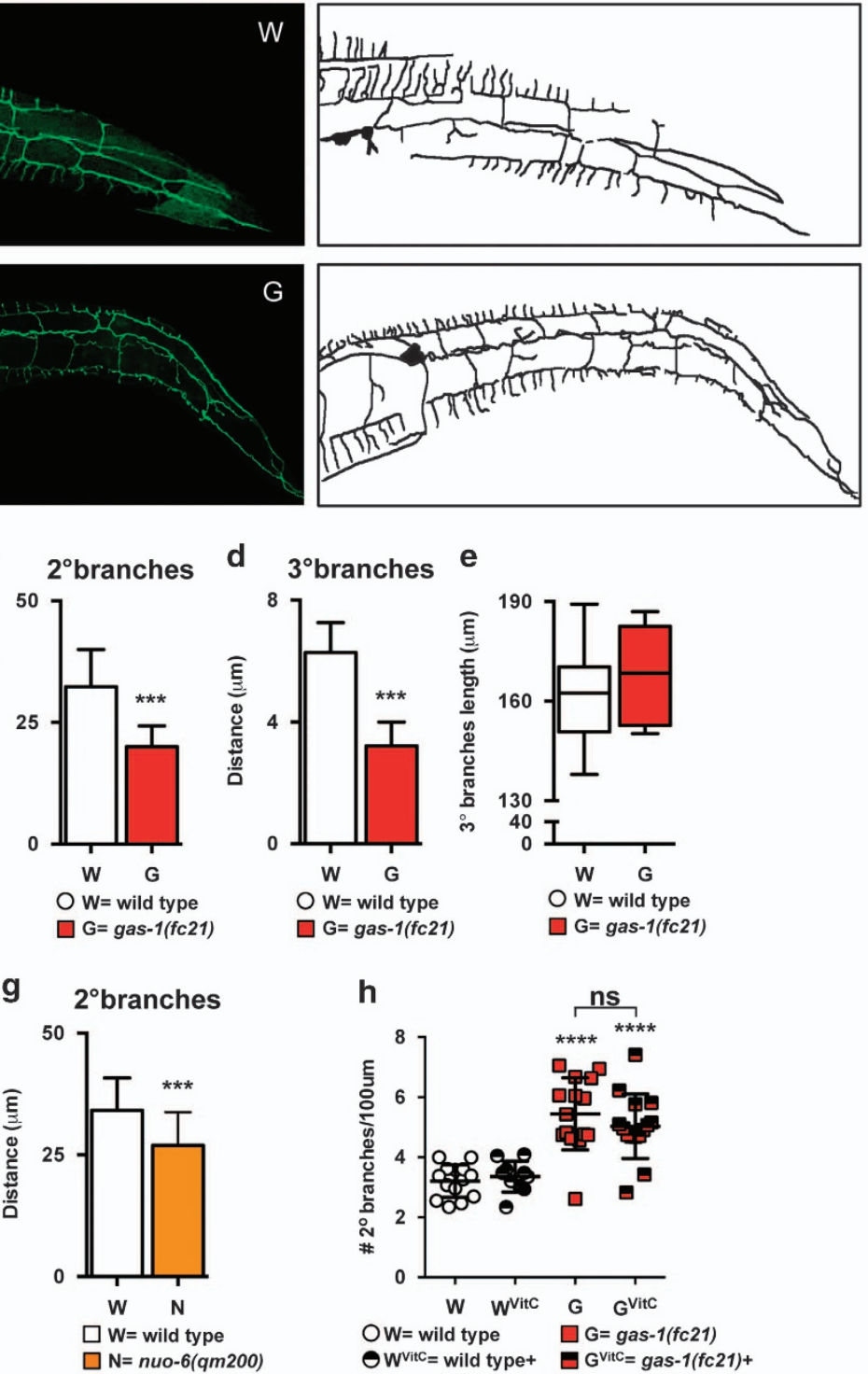

d $3^{\circ}$ branches $\mathrm{e}$
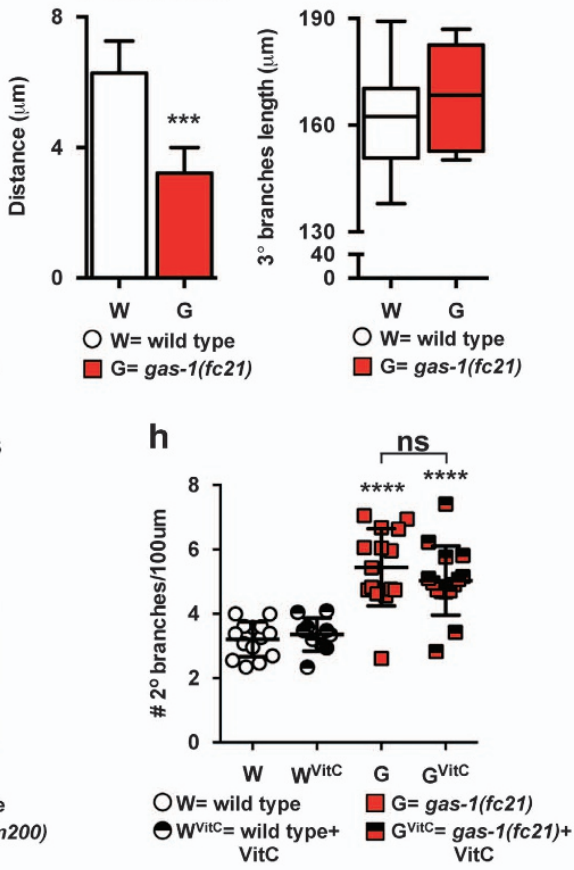
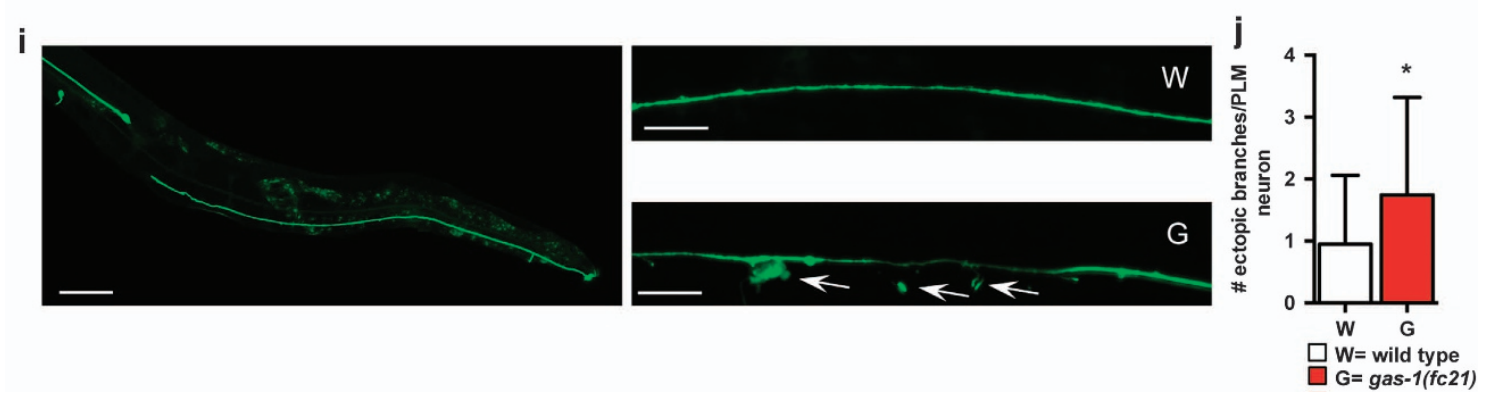

Figure 2 Mitochondrial complex I deficiency increases the branching of $C$. elegans neurons. (a) Confocal images (left panel) and schematic traces (right panel) of wild-type (W) and gas-1 (fc21) (G) mutant nematodes overexpressing the ser-2prom3::gfp transgene. Scale bars represent $25 \mu \mathrm{m}$. (b) Quantification of $2^{\circ}$ branches in wild-type and gas1 mutant nematodes. (c,d) Distance between $2^{\circ}$ (c) and $3^{\circ}$ (d) branches in wild-type and gas-1 mutant nematodes. (e) Quantification of the length of the $3^{\circ}$ branches in wildtype and gas-1 mutant nematodes. (f,g) Quantification of $2^{\circ}$ branches normalized to the body length (f) and the distance between $2^{\circ}$ branches $(\mathbf{g})$ in wild-type and nuo- 6 mutant nematodes. (h) Quantification of $2^{\circ}$ branches normalized to the body length for wild-type and gas-1 mutant nematodes after treatment with $1 \mathrm{mM}$ Vitamin $\mathrm{C}$. (i) Representative confocal image of a wild-type animal (left panel) overexpressing the mec-4p::gfp transgene. Scale bar represents $50 \mu \mathrm{m}$. Representative confocal images (right panel) of a part of the PLM neuron of a wild-type (W) and a gas-1 mutant nematode (G) overexpressing the mec-4p::gfp transgene. The arrows indicate the ectopic processes. Scale bars represent $10 \mu \mathrm{m}$. (j) Quantification of ectopic processes in PLM neurons in wild-type (W) and gas-1 (G) mutants. ${ }^{* * \star *} P<0.0001,{ }^{* * *} P=0.0001$, ${ }^{* *} P<0.001,{ }^{*} P<0.05$, one-way ANOVA (panel H) or Student's $t$-test; $n=3$ for each set of experiment 
a
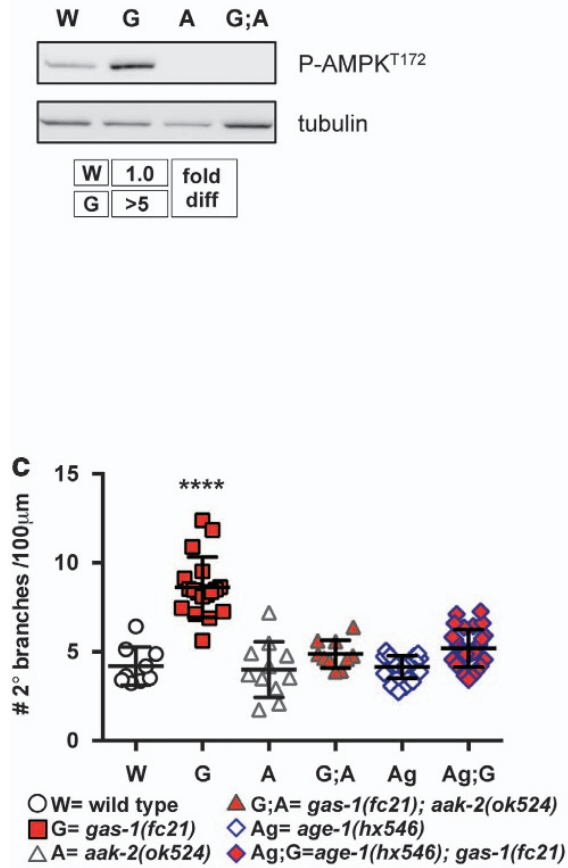

b

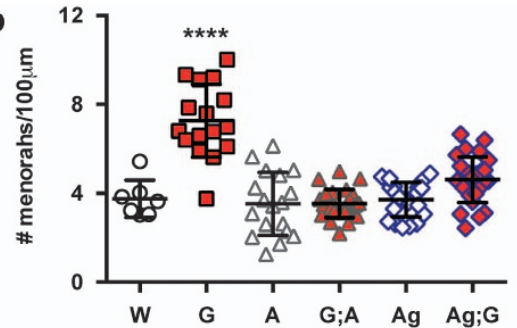

$O W=$ wild type $\quad \Delta G ; A=$ gas-1(fc21); aak-2(ok524) $\square \mathrm{G}=$ gas-1(fc21) $\diamond \mathrm{Ag}=$ age-1(hx546)

$\triangle \mathrm{A}=$ aak-2(ok524) $\diamond \mathrm{Ag} ; \mathrm{G}=$ age-1(hx546); gas-1(fc21)
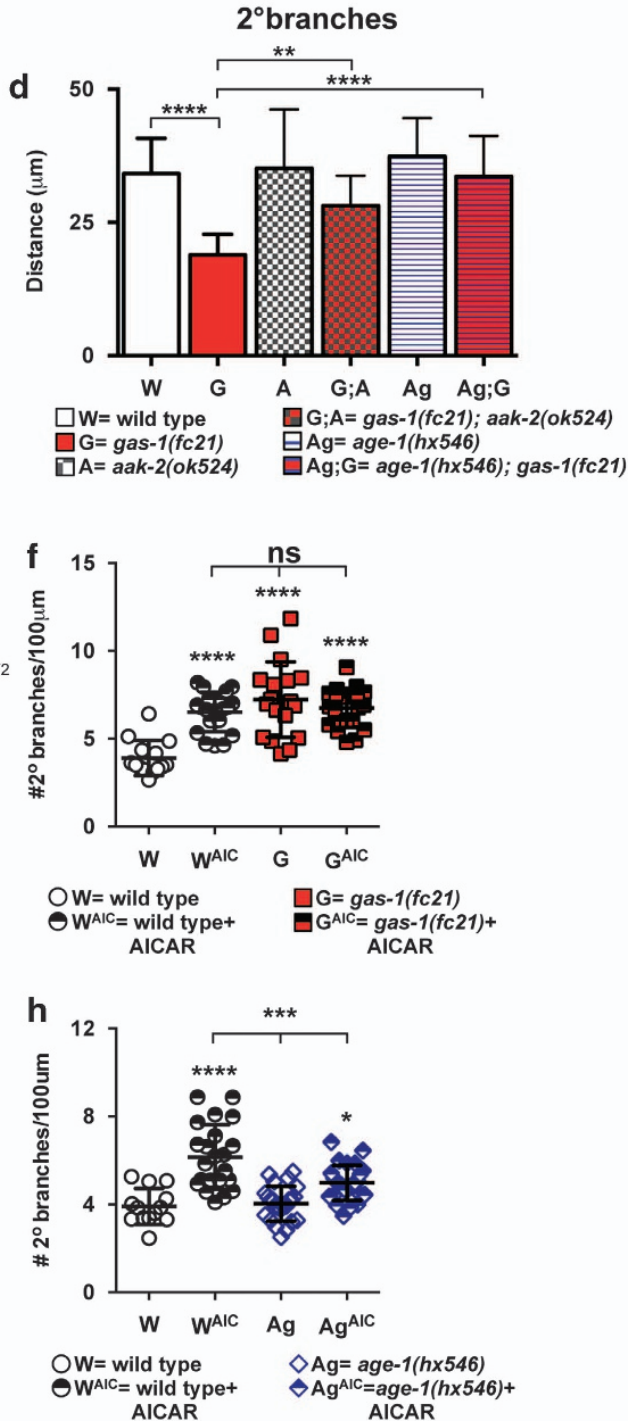

Figure 3 AMPK controls dendritic branching in nematodes with compromised OXPHOS. (a) Immunoblot analysis of phosphorylated AMPK (T172) and tubulin (loading control) in protein extracts from wild-type (W), gas-1 (G), aak-2 (A) and gas-1; aak-2 (G;A) mutant nematodes $(n=1)$. (b,c) Quantification of menorah number (b), number of $2^{\circ}$ branches (c) and distance between $2^{\circ}$ branches (d) in wild-type, gas-1, aak-2, gas-1; aak-2, age-1 and age-1; gas-1 mutant nematodes. The number of menorahs and of ${ }^{\circ}$ branches is normalized to the body length. (e) Immunoblot analysis of phosphorylated AMPK (T172) (tubulin as loading control) for untreated and treated with $1 \mathrm{mM}$ AICAR wild-type (W), gas-1 (G) age-1 (Ag) and aak-2 (A) mutant nematodes $(n=1)$. (f) Number of $2^{\circ}$ branches normalized to the body length in wild type and gas-1 mutant animals untreated or treated with $1 \mathrm{mM}$ AICAR. (g) Number of normalized $2^{\circ}$ branches in aak-2 mutant animals exposed to $1 \mathrm{mM}$ AICAR. (h) Number of $2^{\circ}$ branches normalized to the body length in wild-type and age-1 mutant animals untreated or treated with $1 \mathrm{mM} \mathrm{AICAR} .{ }^{* \star \star *} P<0.0001,{ }^{\star \star \star} P<0.001,{ }^{\star \star} P<0.01,{ }^{*} P<0.05$, one-way ANOVA, $n=3$ (unless otherwise indicated) for each set of experiments and 15-30 animals per condition were analyzed in total

OXPHOS impairment-induced dendritic branching is uncoupled from accelerated aging. Lastly, to rule out that the observed phenotype was simply due to premature aging, we assessed the morphology of PVD neurons in daf-2 mutant animals. Inhibition of the sole insulin/IGF-1 receptor results in enhanced cellular maintenance and prolonged lifespan. ${ }^{24}$ 
Moreover, reduced insulin/IGF-1 signaling suppresses neurite branching. ${ }^{25,26}$ We generated animals overexpressing the ser-2prom3::gfp transgene, carrying the gas-1(fc21) mutation and the hypomorphic allele daf-2(e1370). We observed that the phosphorylation status of $C$. elegans AMPK was significantly higher in the daf-2 background compared with wild type (Figure 4a). Then, we assessed dendrites in daf-2 mutants. Although daf-2; gas-1 double mutants live much longer than daf-2 single mutant and almost three times longer than gas-1(fc21) animals (Troulinaki et al, manuscript in preparation), they displayed the same increased number of $2^{\circ}$ branches accompanied by reduced distance between branches as in gas-1 and daf-2 single mutant nematodes (Figures $4 b$ and $c$ ). This is in line with our previous data in the long-lived nuo-6 mutant nematodes (Figures $2 f$ and $g$ ). Overall, our findings suggest that defective OXPHOS leads to dendritic branching through the modulation of the AMPK/PI3K signaling pathway (Figure 4d). Moreover, they rule out that the increased number of structural units in animals with compromised OXPHOS is the result of premature aging.

\section{Discussion}

Cognitive decline is a common feature of many neurodegenerative disorders and is associated with the loss of neuronal connectivity. In inherited and sporadic cases of human brain pathologies, a persistent age-related impairment of synaptic structures inevitably affects the spatial and temporal neurotransmission between cells, severely compromises the dynamic remodeling of neural circuits and ultimately results in human intellectual disabilities. In this regard, mitochondrial function significantly contributes to the maintenance of both pre- and postsynaptic compartments, thus affecting existing synapses as well as the formation of new ones. ${ }^{27,28}$ Here, we uncover a new signaling cascade that participates in dendritic remodeling as a result of mitochondrial impairment. In animals carrying OXPHOS defects, we provide multiple lines of evidence that illustrate the engagement of the AMPK/PI3K axis and the consequent development of newly formed dendritic branches. Several clues strongly suggest that defined signaling cascades regulate neuronal branching in vivo. For example, the serine threonine kinase LKB1 controls axon specification and branching through the activation of the AMPK-like kinase NUAK1 and the consequent stalling of mitochondria at the nascent presynaptic compartment. ${ }^{29}$ Similarly, AICAR-mediated AMPK activation affects PI3K localization and Akt activity at the neurite tips during development. This initiates a rapid cascade of detrimental signals that inevitably suppresses axonal growth and neuronal polarization. ${ }^{20}$ In our settings, we demonstrated that, in animals carrying mitochondrial defects, OXPHOS inhibition promotes AMPK activity, which then affects the PI3K signaling pathway and the maintenance of the correct dendritic structure.

Beside their role in ATP production, mitochondria are essential in shaping intracellular signaling, especially calcium and cyclic AMP homeostasis. ${ }^{30,31}$ In neurons, intracellular distribution of mitochondria can stimulate the formation of synapses. Although mitochondria are rarely observed within dendritic protrusions, their number critically influences the density, plasticity and turnover of spines. ${ }^{28}$ In response to depolarizing stimuli, mitochondria tend to concentrate particularly in the vicinity of subcellular compartments characterized by high neuronal activity. Most likely, local recruitment of mitochondria underneath the postsynaptic terminus or at the growth cone might provide metabolic support or high a

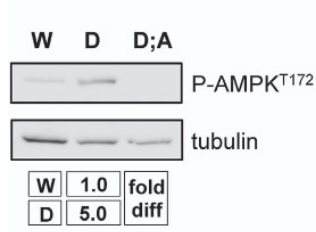

d

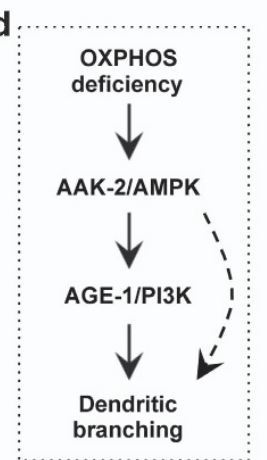

b

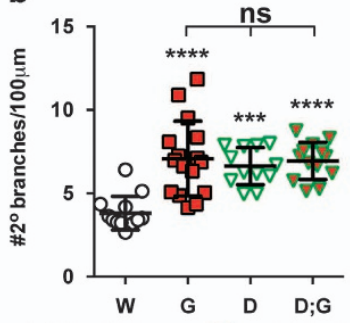

$\square \mathrm{W}=$ wild type $\quad \nabla \mathrm{D}=$ daf-2(e1370) $\square \mathrm{G}=$ gas-1(fc21) $\nabla \mathrm{D} ; \mathrm{G}=$ daf-2(e1370); gas-1(fc21) c $\quad 2^{\circ}$ branches

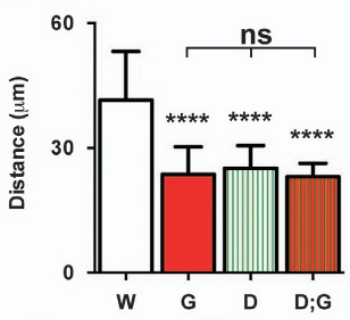

$\square \mathrm{W}=$ wild type $\quad$ 血 $\mathrm{D}=$ daf $-2(\mathrm{e} 1370)$

$\square=$ gas-1(fc21) $\square \mathrm{D} ; \mathrm{G}=$ daf-2(e1370); gas-1(fc21)

Figure 4 Inhibition of the DAF-2/insulin/IGF-1 signaling pathways does not repress OXPHOS impairment-induced branching. (a) Immunoblot analysis of phosphorylated AMPK (T172) and tubulin in samples from wild-type (W), daf-2 (D) and daf-2; aak-2 (D;A) mutant nematodes $(n=1)$. (b,c) Measurement of the number of $2^{\circ}$ branches normalized to the body length (b) and the distance between $2^{\circ}$ branches (c) in wild-type, gas-1, daf-2 and daf-2; gas-1 mutant nematodes $\left(^{* * * *} P<0.0001\right.$, ${ }^{* * *} P<0.001$, $n s=$ not significant, one-way ANOVA, $n=3$ and 15-20 animals per condition were analyzed in total). (d) Schematic summary of the proposed signaling cascade 
calcium buffer capacity. Additionally, in the presence of proper stimulating factors, PI3K-mediated recruitment of mitochondria can stimulate local protein synthesis and, at least in axons, promote branching. ${ }^{32,33}$ Thus, in animals with mitochondrial impairment, it would not be surprising that, through AAK-2/ AMPK and AGE-1/PI3K, changes in mitochondrial numbers or dynamics potentiate dendritic branching. This would be in line with the observation that mitochondria often localize at the branching sites of new dendritic projections. ${ }^{26}$

Previously published works demonstrated that aging severely affects the maintenance of neuronal structures. ${ }^{25,26}$ In agreement with these findings, we confirm that over time PVD neurons exhibit an age-dependent decline of their welldefined spatial organization (data not shown). However, changes in dendritic arbors caused by OXPHOS impairment are not the result of premature aging, as supported by our data in the long-lived daf-2 and nuo- 6 mutant animals. It is intriguing and perhaps counterintuitive that, in impaired energy conditions, $C$. elegans neurons enhance rather than suppress branching. Perhaps sustained activation of essential cellular sensors causes the loss of a well-organized neuronal architecture by inducing the formation of newly generated branches. Alternatively, additional structural components might be a compensatory event due to decreased functionality. Our findings clearly demonstrate a signaling pathway that controls dendritic branching through AMPK/PI3K under conditions of mitochondrial OXPHOS impairment. Previous studies have described the involvement of AMPK, PI3K and mitochondria in axon guidance and growth. ${ }^{20,21,32}$ It is very well possible that the signaling pathway described here might be of relevance also for the remodeling of presynaptic structures and axonal branching under conditions of impaired OXPHOS. Future investigations will be necessary to have a more comprehensive understanding of this important aspect, which is relevant in neurodegenerative disorders associated with mitochondrial deficiency.

\section{Materials and Methods}

Antibodies and reagents. The following antibodies and reagents were used in this study: anti P-AMPK (T172) (Cell Signaling, Danvers, MA, USA), anti-tubulin (SIGMA, St. Louis, MO, USA), HRP-conjugated anti-rabbit and anti-mouse secondary antibodies (Thermo Fisher Scientific, Waltham, MA, USA), AICAR (Toronto Research Chemicals Inc., Toronto, ON, Canada) and Vitamin C (SIGMA).

C. elegans maintenance and strains. Nematodes were maintained according to standard procedures. ${ }^{34}$ AICAR and Vitamin $\mathrm{C}$ were added directly to the nematode growth medium at a final concentration of $1 \mathrm{mM}$. Then, plates were seeded with Escherichia coli OP50 and animals were exposed to the treatment from hatching until the first day of adulthood. The strains used in this study were the following: OH1422 otls138 [ser-2prom3::gfp; rol-6], SK4005 zdls5 [mec-4p::gfp; lin-15(+) (pSK1)], nuo-6(qm200)i; otls138 [ser-2prom3::gfp; rol-6], BAN54 gas-1(fc21)X; otls138 [ser-2prom3::gfp; rol-6], BAN73 daf-2(e1370)III; otls138 [ser-2prom3::gfp; rol-6], BAN74 aak-2(ok524)X; otls138 [ser-2prom3::gfp; rol-6], BAN75 daf-2(e1370)III; gas-1(fc21)X; otls138 [ser-2prom3::gfp; rol-6], BAN83 gas-1(fc21)X; aak-2(ok524)X; otls138 [ser-2prom3::gfp; rol-6], BAN102 gas-1(fc21)X; zdls5 [mec-4::gfp; lin-15(+) (pSK1)], BAN105 age-1(hx546)Il; otls138 [ser-2prom3::gfp; rol-6], BAN117 age-1(hx546)ll; gas-1(fc21)X; otls138 [ser-2prom3::.gfp; rol-6].

Confocal microscopy. Nematodes were synchronized and regularly transferred on freshly seeded plates with $E$. coli OP50 bacteria. At the first day of adulthood, they were mounted on a drop of mounting medium (30\% PEG 8000 in $25 \%$ glycerol), covered with a coverslip and imaged using on a Zeiss LSM700 confocal microscope with a $\times 40$ objective (Carl Zeiss Foundation, Jena, Germany).
Quantitative analysis of sequential images was performed with ZEN software (Carl Zeiss Foundation).

Statistical analysis. Statistical analysis was performed using GraphPad Prism software (Graphpad Software, Inc., La Jolla, CA, USA) and the appropriate statistical test was applied as described. For each group, 15-30 animals were analyzed. Data are mean \pm S.E.M.

\section{Conflict of Interest}

The authors declare no conflict of interest.

Acknowledgements. We thank Ms Christiane Bartling-Kirsch, Ms Gabriele Fleger and Dr. Miriam Jakubik for their technical support. We acknowledge Prof. Sigfriend Hekimi for providing a strain used in this work. We thank many colleagues for their constructive comments. Some strains were provided by the CGC, which is funded by NIH Office of Research Infrastructure Programs (P40 OD010440). Part of the funding for this research was provided by the (DZNE, CIHR, MRC), through the CoEN initiative (www.coen.org).

1. Wallace DC, Fan W, Procaccio V. Mitochondrial energetics and therapeutics. Annu Rev Pathol 2010; 5: 297-348.

2. Koopman WJ, Distelmaier F, Smeitink JA, Willems PH. OXPHOS mutations and neurodegeneration. EMBO J 2012; 32: 9-29.

3. Schagger $\mathrm{H}$, Pfeiffer K. Supercomplexes in the respiratory chains of yeast and mammalian mitochondria. EMBO J 2000; 19: 1777-1783.

4. Lapuente-Brun E, Moreno-Loshuertos R, Acin-Perez R, Latorre-Pellicer A, Colas C, Balsa E et al. Supercomplex assembly determines electron flux in the mitochondrial electron transport chain. Science 2013; 340: 1567-1570.

5. Vafai SB, Mootha VK. Mitochondrial disorders as windows into an ancient organelle. Nature 2012; 491: 374-383.

6. Koopman WJ, Willems PH, Smeitink JA. Monogenic mitochondrial disorders. N Engl J Med 2012; 366: 1132-1141.

7. Zeviani M, Di Donato S. Mitochondrial disorders. Brain 2004; 127: 2153-2172.

8. Troulinaki K, Bano D. Mitochondrial deficiency: a double-edged sword for aging and neurodegeneration. Front Genet 2012; 3: 244.

9. Hekimi S, Lapointe J, Wen Y. Taking a "good" look at free radicals in the aging process. Trends Cell Biol 2011; 21: 569-576.

10. Schon EA, Przedborski S. Mitochondria: the next (neurode)generation. Neuron 2011; 70 : 1033-1053.

11. de Bono M, Maricq AV. Neuronal substrates of complex behaviors in C. elegans. Annu Rev Neurosci 2005; 28: 451-501.

12. Smith CJ, Watson JD, Spencer WC, O'Brien T, Cha B, Albeg A et al. Time-lapse imaging and cell-specific expression profiling reveal dynamic branching and molecular determinants of a multi-dendritic nociceptor in C. elegans. Dev Biol 2010; 345: 18-33.

13. White JG, Southgate E, Thomson JN, Brenner S. The structure of the nervous system of the nematode Caenorhabditis elegans. Philos Trans R Soc Lond B, Biol Sci 1986; 314: $1-340$.

14. Tsalik EL, Niacaris T, Wenick AS, Pau K, Avery L, Hobert O. LIM homeobox gene-dependent expression of biogenic amine receptors in restricted regions of the $C$. elegans nervous system. Develop Biol 2003; 263: 81-102.

15. Kayser EB, Morgan PG, Hoppel CL, Sedensky MM. Mitochondrial expression and function of GAS-1 in Caenorhabditis elegans. J Biol Chem 2001; 276: 20551-20558.

16. Yang W, Hekimi S. Two modes of mitochondrial dysfunction lead independently to lifespan extension in Caenorhabditis elegans. Aging Cell 2010; 9: 433-447.

17. Hardie DG. AMP-activated protein kinase: an energy sensor that regulates all aspects of cell function. Genes Dev 2011; 25: 1895-1908.

18. Apfeld J, O'Connor G, McDonagh T, DiStefano PS, Curtis R. The AMP-activated protein kinase AAK-2 links energy levels and insulin-like signals to lifespan in $C$. elegans. Genes Dev 2004; 18: 3004-3009.

19. Curtis R, O'Connor G, DiStefano PS. Aging networks in Caenorhabditis elegans: AMP-activated protein kinase (aak-2) links multiple aging and metabolism pathways. Aging Cell 2006; 5: 119-126.

20. Amato S, Liu X, Zheng B, Cantley L, Rakic P, Man HY. AMP-activated protein kinase regulates neuronal polarization by interfering with PI 3-kinase localization. Science 2011; 332: 247-251.

21. Chang C, Adler CE, Krause M, Clark SG, Gertler FB, Tessier-Lavigne M et al. MIG-10/ lamellipodin and AGE-1/PI3K promote axon guidance and outgrowth in response to slit and netrin. Curr Biol 2006; 16: 854-862.

22. Friedman DB, Johnson TE. A mutation in the age-1 gene in Caenorhabditis elegans lengthens life and reduces hermaphrodite fertility. Genetics 1988; 118: 75-86.

23. Viscomi C, Bottani E, Civiletto G, Cerutti R, Moggio M, Fagiolari $G$ et al. In vivo correction of COX deficiency by activation of the AMPK/PGC-1alpha axis. Cell Metab 2011; 14: 80-90. 
24. Kenyon C, Chang J, Gensch E, Rudner A, Tabtiang R. A C. elegans mutant that lives twice as long as wild type. Nature 1993; 366: 461-464.

25. Tank EM, Rodgers KE, Kenyon C. Spontaneous age-related neurite branching in Caenorhabditis elegans. J Neurosci 2011; 31: 9279-9288.

26. Toth ML, Melentijevic I, Shah L, Bhatia A, Lu K, Talwar A et al. Neurite sprouting and synapse deterioration in the aging Caenorhabditis elegans nervous system. $J$ Neurosci 2012; 32: 8778-8790

27. Cheng A, Hou Y, Mattson MP. Mitochondria and neuroplasticity. ASN Neuro 2010; 2 : e00045.

28. Li Z, Okamoto K, Hayashi Y, Sheng M. The importance of dendritic mitochondria in the morphogenesis and plasticity of spines and synapses. Cell 2004; 119: 873-887.

29. Courchet J, Lewis TL Jr., Lee S, Courchet V, Liou DY, Aizawa S et al. Terminal axon branching is regulated by the LKB1-NUAK1 kinase pathway via presynaptic mitochondrial capture. Cell 2013; 153: 1510-1525.

30. Rizzuto R, De Stefani D, Raffaello A, Mammucari C. Mitochondria as sensors and regulators of calcium signalling. Nat Rev Mol Cell Biol 2012; 13: 566-578.

31. Di Benedetto G, Scalzotto E, Mongillo M, Pozzan T. Mitochondrial $\mathrm{Ca}(2)(+)$ uptake induces cyclic AMP generation in the matrix and modulates organelle ATP levels. Cell Metab 2013; 17: 965-975.
32. Spillane M, Ketschek A, Merianda TT, Twiss JL, Gallo G. Mitochondria coordinate sites of axon branching through localized intra-axonal protein synthesis. Cell Rep 2013; 5: 1564-1575.

33. Chada SR, Hollenbeck PJ. Mitochondrial movement and positioning in axons: the role of growth factor signaling. J Exp Biol 2003; 206: 1985-1992.

34. Brenner S. The genetics of Caenorhabditis elegans. Genetics 1974; 77: 71-94.

(c) (i) $(9)$ Cell Death and Disease is an open-access journal published by Nature Publishing Group. This work is licensed under a Creative Commons Attribution-NonCommercialNoDerivs 3.0 Unported License. The images or other third party material in this article are included in the article's Creative Commons license, unless indicated otherwise in the credit line; if the material is not included under the Creative Commons license, users will need to obtain permission from the license holder to reproduce the material. To view a copy of this license, visit http://creativecommons.org/licenses/ by-nc-nd/3.0/ 